\title{
ANALYSIS OF AIRCRAFT CLUSTERS TO MEASURE SECTOR-INDEPENDENT AIRSPACE CONGESTION
}

\author{
Karl D. Bilimoria ${ }^{\circ}$ and Hilda Q. Lee \\ NASA Ames Research Center \\ Moffett Field, CA 94035
}

\section{Extended Abstract}

The Distributed Air/Ground Traffic Management (DAG-TM) concept of operations ${ }^{1}$ permits appropriately equipped aircraft to conduct Free Maneuvering operations. These "independent" aircraft have the freedom to optimize their trajectories in real time according to user preferences; however, they also take on the responsibility to separate themselves from other aircraft while conforming to any local Traffic Flow Management (TFM) constraints imposed by the air traffic service provider (ATSP). Examples of local-TFM constraints include temporal constraints such as a required time of arrival (RTA), as well as spatial constraints such as regions of convective weather, special use airspace, and congested airspace.

Under current operations, congested airspace typically refers to a sector(s) that cannot accept additional aircraft due to controller workload limitations; hence Dynamic Density (a metric that is indicative of controller workload) can be used to quantify airspace congestion. However, for Free Maneuvering operations under DAG-TM, an additional metric is needed to quantify the airspace congestion problem from the perspective of independent aircraft. Such a metric would enable the ATSP to prevent independent aircraft from entering any local areas of congestion in which the flight deck based systems and procedures may not be able to ensure separation. This new metric, called Gaggle Density, ${ }^{1}$ offers the ATSP a mode of control to regulate normal operations and to ensure safety and stability during rare-normal or off-normal situations (e.g., system failures). It may be difficult to certify Free Maneuvering systems for unrestricted operations, but it may be easier to certify systems and procedures for specified levels of Gaggle Density that could be monitored by the ATSP, and maintained through relatively minor flow-rate (RTA type) restrictions.

Since flight deck based separation assurance is airspace independent, the challenge is to measure congestion independent of sector boundaries. Figure 1, reproduced from Ref. 1, depicts an example traffic situation. When the situation is analyzed by sector boundaries (left side of figure), a Dynamic Density metric would identify excessive congestion in the central sector. When the same traffic situation is analyzed independent of sector boundaries (right side of figure), a Gaggle Density metric would identify congestion in two dynamically defined areas covering portions of several sectors. The first step towards measuring airspace-independent congestion is to identify aircraft clusters, i.e., groups of closely spaced aircraft. The objective of this work is to develop techniques to detect and classify clusters of aircraft.

\footnotetext{
${ }^{\circ}$ Research Scientist, Automation Concepts Research Branch, Mail Stop 210-10; Karl.Bilimoria@nasa.gov. Associate Fellow, AIAA.

- Senior Programmer/Analyst, University of California - Santa Cruz.
} 

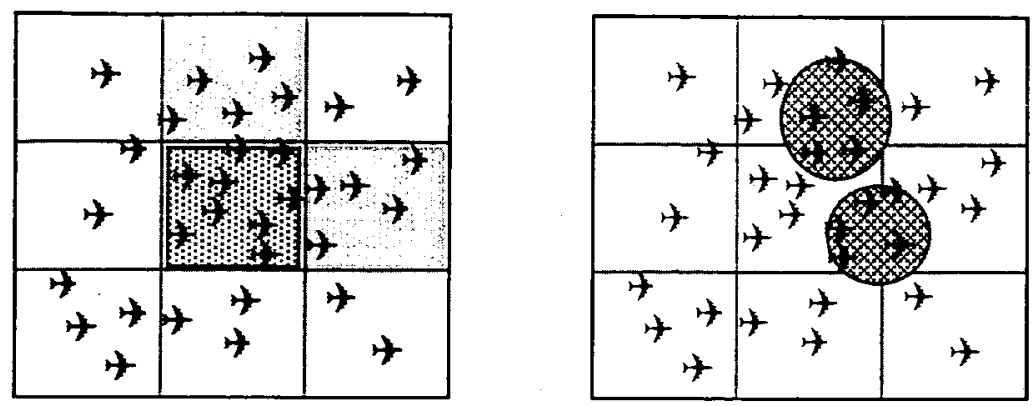

Figure 1: Dynamic Density (left) vs. Gaggle Density (right)

This work adapts clustering techniques that have been successfully utilized for computer vision $^{2}$ applications, e.g., local techniques such as blob coloring, global techniques such as region growing, and other splitting/merging techniques. A simple clustering technique adapted to the air traffic problem is proposed, as outlined below:

1. Set a cluster threshold distance $D_{C T}$.

2. Randomly pick an aircraft $A^{0}$, and identify all aircraft $A_{i}^{1}$ within distance $D_{C T}$ from $A^{0}$.

3. Identify all aircraft $A_{i}^{2}$ within distance $D_{C T}$ from each aircraft $A_{i}^{1}$.

4. Repeat until no more aircraft proximate aircraft can be identified. All proximate aircraft that have been identified $\left(A^{0}, A_{i}^{1}, A_{i}^{2}, \ldots, A_{i}^{n}\right)$ are assigned to the first cluster $C_{1}$.

5. Randomly pick an aircraft not assigned to $C_{1}$, and repeat the process to obtain $C_{2}, C_{3}$, etc.

6. Any aircraft not assigned to a cluster are categorized as a group of sparse background traffic.

This technique has being implemented and is currently being evaluated using recorded track data from the Cleveland Center. An iterative algorithm has been developed for determining appropriate values of $D_{C T}$ to accurately identify aircraft clusters in high-density en route airspace. This algorithm first identifies "natural neighbors" via Delaunay Triangulation, ${ }^{3}$ and then utilizes a clustering metric to determine the best value of $D_{C T}$. Details of the algorithm and numerical results will be presented in the final version of the full paper.

\section{References}

1. Green, S.M., Bilimoria, K.D., and Ballin, M.G., "Distributed Air/Ground Traffic Management for En Route Flight Operations," Air Traffic Control Quarterly, Vol. 9, No. 4, 2001, pp. 259-285.

2. Ballard, D.H. and Brown, C.M., Computer Vision, Prentice-Hall, Englewood Cliffs, NJ, 1982, pp. 149-165.

3. Preparata, F. and Shamos, M.I., Computational Geometry, Springer Verlag, New York, 1985. 